\title{
Role Adaptation of Human and Robot in Collaborative Tasks
}

\author{
Yanan Li, Keng Peng Tee, Wei Liang Chan, Rui Yan, Yuanwei Chua, and Dilip Kumar Limbu
}

\begin{abstract}
In this paper, a role adaptation method is developed for human-robot collaboration based on game theory. This role adaptation is engaged whenever the interaction force changes, causing the proportion of control sharing between human and robot to vary. In one boundary condition, the robot takes full control of the system when there is no human intervention. In the other boundary condition, it becomes a follower when the human exhibits strong intention to lead the task. Experimental results show that the proposed method yields better overall performance than fixed-role interactions.
\end{abstract}

\section{INTRODUCTION}

Human and robot have complementary capabilities, and their collaboration is essential in many situations [1]. However, the design and analysis of their interactive behavior is challenging due to the need to consider not only stability, but also safety and intuitiveness [2]. While techniques to deal with multi-agent collaboration are abundant (e.g. [3], [4]), many of them consider robots only and cannot be applied when humans are in the loop, since the critical issues of uncertainty and unobservable states were not addressed [5].

In the early literature of human-robot interaction, many works adopted conservative approaches of assigning a passive follower role to the robot, e.g., impedance control [6]. Based on the fixed-role leader-follower paradigm, various recognition and estimation methods have been developed (see e.g. [7], [8]) to help the robot understand human intention and interact with its human partner. The underlying assumption of these fixed-role approaches is that humans, having better situational awareness and decision-making skills, should always lead, while robots should always yield and assist humans in tasks requiring high precision and strength. This paradigm works very well for some applications, but it is questionable whether this paradigm can generalize to other applications. For example, in a collaborative welding task, it may be more beneficial for a robot to lead during the welding process since it can sense the process states or positioning errors more accurately than a human. Similar ideas are adopted in [9], where the so-called "obstacle avoidance" problem is studied, and the leading role is given to the robot instead of the human whenever the human-driven motion has an impending collision. Also, the requirement for the human to lead all the time may induce high cognitive load and may not provide optimal performance under prolonged usage.

\footnotetext{
*This work is supported by Grant No. 1225100001 from the Science and Engineering Research Council (SERC), A*STAR, Singapore.

Y. Li, K. P. Tee, W. L. Chan, R. Yan, Y. Chua, and D. K. Limbu are with the Institute for Infocomm Research (I2R), Agency for Science, Technology and Research (A*STAR), Singapore 138632. $\{1$ iy, kptee, chanwl, ryan, ychua, dklimbu\} Qi2r.a-star.edu.sg
}

In [10], it has been highlighted that a framework with adjustable leader/follower roles is essential for human-robot joint motor actions. Risk-sensitive optimal feedback control is proposed in [11], which is the basis of the following two works: in [12], role adaptation is achieved by adaptive attitude design depending on both the disagreement level and environmental situation; and in [13], an adaptation strategy is developed to switch between model-based and model-free predictions in the case of partially known tasks. In [14], a shared plan of human and the robot is given as a desired trajectory of the object configuration and the common goal of the cooperation is known to both agents. In [15], impedance parameters of the human arm are estimated online, and the leader/follower role of robot is switched according to the recognized human intention. This method considers only the extreme cases where the robot is either a leader or a follower. In [16], a homotopy switching model is proposed to switch leader/follower roles in dyadic haptic collaborative tasks through an intermediate object.

Game theory provides useful tools to analyze complex interactive behaviors involving multiple agents. Inspired by the insights from [17], we employ game theory to analyze human-robot interaction, and develop a specific framework which can be implemented in practical robot control. Besides this, we propose an adaptation law that automatically adjusts the role of the robot based on the measured interaction force, in order to achieve human-robot coordination. The proposed role adaptation is continuous instead of switching between different discrete states, and it does not require the common goal known to both agents.

The rest of this paper is organized as follows. In Section II, system description and problem formulation are discussed. In Section III, human-robot collaboration is analyzed by game theory for two-agent games and an updating law is developed for role adaptation. In Section IV, the performance of the proposed method is illustrated through experimental studies. Section V concludes this work.

\section{PROBLEM FORMULATION}

In this paper, we consider a scenario where a robot collaborates with a human to perform a task on a workpiece (e.g. painting or welding). In this scenario, leadership should tend towards the robot when its end-effector is near the workpiece, since the robot is able to sense the workpiece more accurately and perform more precise motion control. Conversely, as the robot end-effector moves further away from the workpiece, the leading role should tend towards the human to increase flexibility in dealing with unexpected problems that may arise, especially in unstructured environments. 
The forward kinematics of the robot are given by $x(t)=$ $\phi(q(t))$, where $x(t) \in \mathbb{R}^{m}$ and $q(t) \in \mathbb{R}^{n}$ are positions in the Cartesian space and the joint space, respectively. Differentiating it with respect to time results in $\dot{x}(t)=$ $J(q(t)) \dot{q}(t)$, where $J(q(t)) \in \mathbb{R}^{m \times n}$ is the Jacobian matrix. The dynamics of the robot in the joint space are given by

$$
\begin{aligned}
& M(q(t)) \ddot{q}(t)+C(q(t), \dot{q}(t)) \dot{q}(t)+G(q(t)) \\
& =\tau(t)+J^{T}(q(t)) f(t)
\end{aligned}
$$

where $M(q(t)) \in \mathbb{R}^{n \times n}$ denotes the inertia matrix, $C(q(t), \dot{q}(t)) \dot{q}(t) \in \mathbb{R}^{n}$ the Coriolis and centrifugal forces, $G(q(t)) \in \mathbb{R}^{n}$ the gravitational force, $\tau(t) \in \mathbb{R}^{n}$ the control input, and $f(t) \in \mathbb{R}^{n}$ the interaction force applied by the human.

The robot is under impedance control which is implemented using two loops: an inner position control loop and an outer loop [18]. As state-of-the-art robots have controllers that provide very accurate joint position control, we assume a perfect inner position control loop, i.e., $q(t)=q_{r}(t)$, where $q_{r}(t)$ is the reference angle/position. The outer loop is used to generate $q_{r}(t)$ according to the following impedance model in the Cartesian space:

$$
M_{d} \ddot{x}_{r}(t)+C_{d} \dot{x}_{r}(t)=u(t)+f(t)
$$

where $M_{d} \in \mathbb{R}^{m \times m}$ and $C_{d} \in \mathbb{R}^{m \times m}$ are given inertial and damping matrices, respectively, $u(t) \in \mathbb{R}^{m}$ is the control input of the robot in the Cartesian space, and $x_{r}(t) \in \mathbb{R}^{m}$ is the reference position of the robot in the Cartesian space. By designing $u(t)$ and measuring $f(t)$, the reference velocity is obtained based on inverse kinematics, i.e., $\dot{q}_{r}(t)=J^{\dagger}(q) \dot{x}_{r}(t)$, where $J^{\dagger}(q)$ is the pseudo-inverse of the Jacobian matrix $J(q)$. Based on the above assumption of a perfect inner position control loop, we have

$$
M_{d} \ddot{x}(t)+C_{d} \dot{x}(t)=u(t)+f(t)
$$

Without loss of generality, we assume that the human position is also $x(t)$. From (3), we see that the two sources $u(t)$ and $f(t)$ are sharing control of the robot.

As there are two control inputs from human and robot, we consider the following finite-horizon cost function:

$$
\begin{aligned}
\Gamma= & \int_{0}^{t_{f}}\left(\left(x-x_{d}\right)^{T} Q_{1}\left(x-x_{d}\right)+\dot{x}^{T} Q_{2} \dot{x}+u^{T} R_{1} u\right. \\
& \left.+f^{T} R_{2} f\right) d t
\end{aligned}
$$

where $t_{f}$ is the terminal time, $x_{d}$ the desired trajectory of the robot, and $Q_{1}, Q_{2} \in \mathbb{R}^{m \times m} \geq 0, R_{1}, R_{2} \in \mathbb{R}^{m \times m}>0$ are weights. The first term penalizes the error between the actual position and the desired position of the robot, while the second term makes the robot's motion smooth. The last two terms determine the contributions of robot and human: a larger $R_{1}$ indicates that human has a more leading role, and conversely, a larger $R_{2}$ indicates that robot has a more leading role. Due to the existence of uncertainties in the environment (which includes humans), the issues of how to parameterize the cost function (4), and how to update it onthe-fly, are challenging [19]. It is the aim of this work to (at least partially) address these issues.

\section{ROLE ADAPTATION}

\section{A. Two-Player Game}

From the aforementioned problem statement, we realize that the human-robot collaboration problem can be understood from the point of view of game theory. In game theory, different types of multi-agent behaviors have been analyzed, such as cooperation and competition [20], [21]. Besides, there are different optimization criteria which will result in different multi-agent behaviors, and herein we consider the Nash equilibrium.

For ease of analysis using game theory, Equ. (3) is rewritten in the following state-space form

$$
\dot{z}(t)=A z(t)+B_{1} u(t)+B_{2} f(t)
$$

where $z(t)=\left[x^{T}(t) \dot{x}^{T}(t)\right]^{T}, A=\left[\begin{array}{cc}\mathbf{0}_{m \times m} & I_{m \times m} \\ \mathbf{0}_{m \times m} & -M_{d}^{-1} C_{d}\end{array}\right]$, and $B_{1}=B_{2}=\left[\begin{array}{c}\mathbf{0}_{m \times m} \\ M_{d}^{-1}\end{array}\right]$, with $\mathbf{0}_{m \times m}$ and $I_{m \times m}$ denoting $m \times m$ zero and unit matrices, respectively. Besides, the optimal tracking problem as discussed in Section II needs to be transformed into a regulation problem [22]. In particular, the desired trajectory of the robot $x_{d}$ is generated by the following system

$$
\left\{\begin{array}{l}
\dot{w}=U w \\
x_{d}=V w
\end{array}\right.
$$

where $w \in \mathbb{R}^{l}$ is an auxiliary state, and $U \in \mathbb{R}^{l \times l}$ and $V \in$ $\mathbb{R}^{n \times l}$ are two design matrices. Then, with the augmented state $\bar{z}=\left[z^{T} w^{T}\right]^{T}$, we have the following system

$$
\dot{\bar{z}}=\bar{A} \bar{z}+\bar{B}_{1} u+\bar{B}_{2} f
$$

where $\bar{A}=\left[\begin{array}{cc}A & \mathbf{0}_{2 m \times l} \\ \mathbf{0}_{l \times 2 m} & U\end{array}\right]$ and $\bar{B}_{1}=\bar{B}_{2}=\left[\begin{array}{c}B_{1} \\ \mathbf{0}_{l \times m}\end{array}\right]$, with $\mathbf{0}_{2 m \times l}, \mathbf{0}_{l \times 2 m}$, and $\mathbf{0}_{l \times m}$ denoting zero matrices with proper dimensions. The cost function is thus rewritten as

$$
\Gamma=\int_{0}^{t_{f}}\left(\bar{z}^{T} Q \bar{z}+u^{T} R_{1} u+f^{T} R_{2} f\right) d t
$$

where $Q=\left[\begin{array}{ccc}Q_{1} & \mathbf{0}_{m \times m} & -Q_{1} V \\ \mathbf{0}_{m \times m} & Q_{2} & \mathbf{0}_{m \times l} \\ -V^{T} Q_{1} & \mathbf{0}_{l \times m} & V^{T} Q_{1} V\end{array}\right]$.

Based on game theory, the human partner also has a cost function to describe his/her objective, which is, however, unknown to the robot. In this regard, a solution of Nash equilibrium cannot be obtained. To cope with this problem, we develop an approach to update the robot's role such that it coordinates with its human partner, which is detailed in the following subsection.

\section{B. Adaptation Law: Towards Coordination}

Suppose that the cost function of the human is also $\Gamma$ as in (8). It indicates that both human and robot have a common objective, which is referred to as "coordination" [20], [21]. In 
this case, the Nash equilibrium is achieved by the following optimal control:

$$
\begin{aligned}
u^{*} & =-\frac{1}{2} R_{1}^{-1} \bar{B}_{1}^{T} P \bar{z}^{*} \\
f^{*} & =-\frac{1}{2} R_{2}^{-1} \bar{B}_{2}^{T} P \bar{z}^{*}
\end{aligned}
$$

where $P$ is obtained from the following Riccati equation

$$
\begin{aligned}
& \bar{A}^{T} P+P \bar{A}+Q-P \bar{B}_{1} R_{1}^{-1} \bar{B}_{1}^{T} P \\
& -P \bar{B}_{2} R_{2}^{-1} \bar{B}_{2}^{T} P=\mathbf{0}_{m \times m}
\end{aligned}
$$

and $\bar{z}^{*}$ is the optimal state in the following optimal system

$$
\dot{\bar{z}}^{*}=\bar{A} \bar{z}^{*}+\bar{B}_{1} u^{*}+\bar{B}_{2} f^{*}
$$

However, as mentioned above, the cost function of the human partner is unknown and probably not $\Gamma$, which results in different Nash equilibria requiring control inputs different from (9) and (10). As the actual control input of the human partner, $f$, is measurable, its difference from $f^{*}$ can be deemed as a measure of conflict between the human partner's objective and current situation. In this regard, this difference can be used to adjust the role of the robot.

Since the relative roles of human and robot are determined by $R_{1}$ and $R_{2}$ in the cost function (4), we may update either $R_{1}$ or $R_{2}$ to minimize the following force error

$$
E=\frac{1}{2} e_{f}^{T} e_{f}
$$

where $e_{f}=f-f^{*}$. This can be realized by

$$
\dot{R}_{2}=-\alpha \frac{\partial E}{\partial R_{2}}
$$

where $\alpha>0$ is the updating rate. For simplicity of implementation, we assume that $R_{2}=r_{2} I_{m \times m}$. Then, we obtain a simplified role adaptation law as below

$$
\dot{r}_{2}=-\alpha \frac{\partial E}{\partial r_{2}}=-\alpha e_{f}^{T} \frac{\partial e_{f}}{\partial r_{2}}
$$

According to Equ. (10), we have

$$
\frac{\partial e_{f}}{\partial r_{2}}=\frac{1}{2 r_{2}^{2}} \bar{B}_{2}^{T} P \bar{z}^{*}-\frac{1}{2 r_{2}} \bar{B}_{2}^{T} \frac{\partial P}{\partial r_{2}} \bar{z}^{*}-\frac{1}{2 r_{2}} \bar{B}_{2}^{T} P \frac{\partial \bar{z}^{*}}{\partial r_{2}}
$$

While $P(t)$ is obtained by solving Equ. (11), $\frac{\partial P}{\partial r_{2}}$ is obtained by solving the following equation

$$
\begin{array}{r}
\bar{A}^{T} \frac{\partial P}{\partial r_{2}}+\frac{\partial P}{\partial r_{2}} \bar{A}-\frac{\partial P}{\partial r_{2}} \bar{B}_{1} R_{1}^{-1} \bar{B}_{1}^{T} P \\
-P \bar{B}_{1} R_{1}^{-1} \bar{B}_{1}^{T} \frac{\partial P}{\partial r_{2}}-\frac{\partial P}{\partial r_{2}} \bar{B}_{2} R_{2}^{-1} \bar{B}_{2}^{T} P \\
-P \bar{B}_{2} R_{2}^{-1} \bar{B}_{2}^{T} \frac{\partial P}{\partial r_{2}}+\frac{1}{r_{2}^{2}} P \bar{B}_{2} \bar{B}_{2}^{T} P=0
\end{array}
$$

which is obtained by differentiating (11) with respect to $r_{2}$. By denoting $X=\bar{A}-\left(\bar{B}_{1} R_{1}^{-1} \bar{B}_{1}^{T}+\bar{B}_{2} R_{2}^{-1} \bar{B}_{2}^{T}\right) P$ and $Y=-\frac{1}{r_{2}^{2}} P \bar{B}_{2} \bar{B}_{2}^{T} P$, we rewrite (17) as $X^{T} \frac{\partial P}{\partial r_{2}}+\frac{\partial P}{\partial r_{2}} X=Y$, for which the solution is immediately obtained as $\frac{\partial P}{\partial r_{2}}=$ $\frac{1}{2} X^{-T} Y$, considering that $\frac{\partial P}{\partial r_{2}}$ and $Y$ are symmetric. Besides, we have

$$
\dot{\bar{z}}^{*}=\dot{r}_{2} \frac{\partial \bar{z}^{*}}{\partial r_{2}}
$$

which can be obtained from (12). Substituting (15) and (16) into (18), we obtain $\frac{\partial \bar{z}^{*}}{\partial r_{2}}$, and thus $\frac{\partial e_{f}}{\partial r_{2}}$ in (16). After $R_{2}$ is updated and $P$ obtained, the actual control of the robot is obtained as follows

$$
u=-\frac{1}{2} R_{1}^{-1} \bar{B}_{1}^{T} P \bar{z}
$$

\section{Interpretation}

Interpreting the role adaptation law (14), the robot adjusts its role according to the new role of the human. Specifically, whenever the human interacts with the robot by exerting a force $f$, the weight $R_{2}$ will be updated via (14), until $f$ tracks the optimal control $f^{*}$. Denote $K_{1}$ and $K_{2}$ as the two feedback gain matrices for optimal robot control and human control: $K_{1}=\frac{1}{2} R_{1}^{-1} \bar{B}_{1}^{T} P$ and $K_{2}=\frac{1}{2} R_{2}^{-1} \bar{B}_{2}^{T} P$, respectively. It is noted that $K_{1}$ will be updated during the role adaptation due to the change of $P$. By substituting $u=$ $-K_{1} \bar{z}$ into the impedance model (3), we obtain

$$
M_{d} \ddot{x}(t)+C_{d} \dot{x}(t)+K_{1} \bar{z}=f
$$

Suppose that $K_{1}=\left[\begin{array}{lll}K_{1,1} & K_{1,2} & K_{1,3}\end{array}\right]$, where $K_{1,1} \in \mathbb{R}^{m \times m}$, $K_{1,2} \in \mathbb{R}^{m \times m}$, and $K_{1,3} \in \mathbb{R}^{m \times l}$. Then, the above equation becomes

$$
M_{d} \ddot{x}(t)+\left(C_{d}+K_{1,1}\right) \dot{x}(t)+\left(K_{1,2} x+K_{1,3} w\right)=f
$$

It can be found that $K_{1,1}$ is used to modulate the damping of the given impedance model, while $\left(K_{1,2} x+K_{1,3} w\right)$ is the stiffness component which takes the desired trajectory of the robot $x_{d}$ (recalling (6)) into consideration. From this point of view, the resulting control $u$ is a variable impedance control which simultaneously modulates damping and stiffness [23], and incorporates the desired trajectory of the robot.

\section{Stability Analysis}

Subtracting (12) from (7), we obtain $\dot{e}_{\bar{z}}=\bar{A} e_{\bar{z}}+\bar{B}_{1}(u-$ $\left.u^{*}\right)+\bar{B}_{2} e_{f}$, where $e_{\bar{z}}=\bar{z}-\bar{z}^{*}$. Considering optimal control (9) and actual control (19), we have the following closedloop error dynamics

$$
\dot{e}_{\bar{z}}=\left(\bar{A}-\bar{B}_{1} K_{1}\right) e_{\bar{z}}+\bar{B}_{2} e_{f}
$$

Theorem 1: Consider the robot dynamics described by the impedance model (3). If $f$ is of class $C^{2}$, the control input (19) with the role adaptation law (15) will guarantee that

- $\lim _{t \rightarrow \infty} e_{f}(t)=0$, which indicates that optimal control from the human side is achieved in the sense that the cost function (4) is minimized;

- $\lim _{t \rightarrow \infty} u(t)=u^{*}(t)$, which indicates that optimal control from the robot side is achieved; and

- all the other closed-loop signals are bounded.

Proof: Consider a Lyapunov function candidate

$$
W=E+\frac{\chi}{2} e_{\bar{z}}^{T} e_{\bar{z}}
$$

where $\chi=\frac{4 \alpha \lambda_{1} \lambda_{2}}{\left\|B_{2}\right\|^{2}}$, with $\lambda_{1}$ being the lower bound of the minimum eigenvalue of $\frac{\partial e_{f}}{\partial r_{2}}\left(\frac{\partial e_{f}}{\partial r_{2}}\right)^{T}$ and $\lambda_{2}$ the minimum eigenvalue of $\bar{B}_{1} K_{1}-\bar{A}$. 
Differentiating (23) with respect to time, and considering (15) and (22), we obtain

$$
\begin{aligned}
\dot{W}= & \left(\frac{\partial E}{\partial r_{2}}\right)^{T} \dot{r}_{2}+\chi e_{\bar{z}}^{T} \dot{e}_{\bar{z}} \\
= & -\alpha e_{f}^{T} \frac{\partial e_{f}}{\partial r_{2}}\left(\frac{\partial e_{f}}{\partial r_{2}}\right)^{T} e_{f}+\chi e_{\bar{z}}^{T}\left(\bar{A}-\bar{B}_{1} K_{1}\right) e_{\bar{z}} \\
& +\chi e_{\bar{z}}^{T} \bar{B}_{2} e_{f} \\
\leq & -\alpha \lambda_{1}\left\|e_{f}\right\|^{2}-\chi \lambda_{2}\left\|e_{\bar{z}}\right\|^{2}+\chi e_{\bar{z}}^{T} \bar{B}_{2} e_{f} \\
= & -\left(\sqrt{\alpha \lambda_{1}}\left\|e_{f}\right\|-\sqrt{\chi \lambda_{2}}\left\|e_{\bar{z}}\right\|\right)^{2} \\
& -2 \sqrt{\alpha \chi \lambda_{1} \lambda_{2}}\left\|e_{f}\right\|\left\|e_{\bar{z}}\right\|+\chi e_{\bar{z}}^{T} \bar{B}_{2} e_{f} \\
\leq & \left(-2 \sqrt{\alpha \chi \lambda_{1} \lambda_{2}}+\chi\left\|\bar{B}_{2}\right\|\right)\left\|e_{f}\right\|\left\|e_{\bar{z}}\right\|=0
\end{aligned}
$$

Hence, if $\lambda_{1} \neq 0, e_{f}$ and $e_{\bar{z}}$ are bounded; otherwise, Equ. (15) indicates $\dot{r}_{2}=0$, and thus $e_{f}$ and $e_{\bar{z}}$ are bounded. According to (22), $\dot{e}_{\bar{z}}$ is bounded.

Because the system under study is not autonomous, we verify the boundedness of $\ddot{W}$ to conclude the asymptotic stability, according to Barbalat's lemma [24]. Further differentiating (24) leads to

$$
\ddot{W}=e_{f}^{T} \ddot{e}_{f}+\dot{e}_{f}^{T} \dot{e}_{f}+e_{\bar{z}}^{T} \ddot{e}_{\bar{z}}+\dot{e}_{\bar{z}}^{T} \dot{e}_{\bar{z}}
$$

Thus, in order to show that $\ddot{W}$ is bounded, we need to show that the following signals are bounded: $\dot{e}_{f}, \ddot{e}_{f}$, and $\ddot{e}_{\bar{z}}$.

Since $f$ is exerted by the human partner and it is bounded, $f^{*}$ is bounded. Comparing (10) and (9) and considering (11), we obtain that $u^{*}$ is bounded, as well as $P$ and $\dot{P}$. Hence, $\dot{r}_{2}$ is bounded according to (15), and $\bar{z}^{*}$ and $\dot{\bar{z}}^{*}$ are bounded according to (12). Considering (10), we have

$$
\dot{e}_{f}=\dot{f}-\frac{\dot{r}_{2}}{2 r_{2}} \bar{B}_{2}^{T} P \bar{z}^{*}+\frac{1}{2 r_{2}} \bar{B}_{2}^{T} \dot{P} \bar{z}^{*}+\frac{1}{2 r_{2}} \bar{B}_{2}^{T} P \dot{\bar{z}}^{*}
$$

Since $\dot{f}$ is bounded, $\dot{e}_{f}$ is bounded. Further differentiating the above equation leads to

$$
\begin{aligned}
\ddot{e}_{f}= & \ddot{f}-\frac{\ddot{r}_{2}}{2 r_{2}^{2}} \bar{B}_{2}^{T} P \bar{z}^{*}-\frac{\dot{r}_{2}}{2 r_{2}^{2}} \bar{B}_{2}^{T} \dot{P} \bar{z}^{*}-\frac{\dot{r}_{2}}{2 r_{2}^{2}} \bar{B}_{2}^{T} P \dot{\bar{z}}^{*} \\
& +\frac{1}{2 r_{2}} \bar{B}_{2}^{T} \ddot{P} \bar{z}^{*}+\frac{1}{r_{2}} \bar{B}_{2}^{T} \dot{P}^{*} \dot{\bar{z}}^{*}+\frac{1}{2 r_{2}} \bar{B}_{2}^{T} P \ddot{\bar{z}}^{*}
\end{aligned}
$$

In the above equation, $\ddot{r}_{2}$ is bounded by differentiating (15), and $\ddot{P}$ is bounded by differentiating (11). Considering (9), $\dot{u}^{*}$ is bounded. By differentiating (12), we have that $\ddot{\bar{z}}^{*}$ is bounded. Hence, $\ddot{e}_{f}$ is bounded. Besides, $\ddot{e}_{\bar{z}}$ is bounded by differentiating (22).

Therefore, we conclude that $\ddot{W}$ is bounded. According to Barbalat's lemma [24], we claim that $\lim _{t \rightarrow \infty} e_{f}(t)=0$ and $\lim _{t \rightarrow \infty} e_{\bar{z}}(t)=0$, which lead to $\lim _{t \rightarrow \infty} u(t)=u^{*}(t)$, by considering the control input (19).

\section{EXPERIMENT}

We consider a scenario sketched in Fig. 1, where a human hand holds the end-effector of a robot. One potential application of this scenario is robotic welding: while the robot has a prescribed trajectory with a basic shape (e.g., circle or straight line), the human partner may have his/her own desired path based on the actual odd shape of the workpiece.

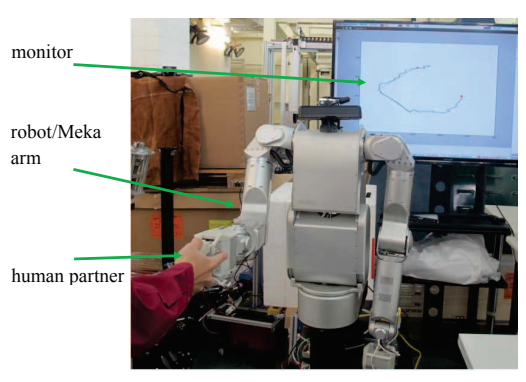

Fig. 1. Experiment setup
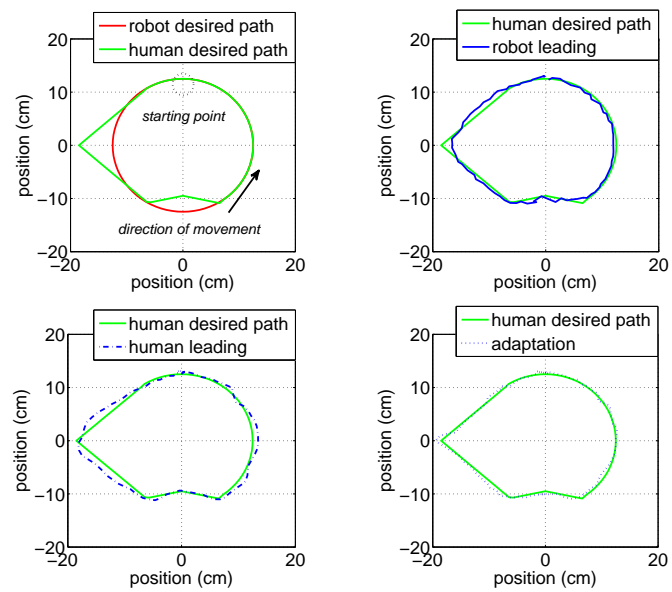

Fig. 2. Trajectories for adaptive and fixed role cases

A 7-DOF Meka A1 arm is used as the experiment platform, of which each joint is under position control. The joint position is measured directly by the ContElec Vert-X 13 encoder at the joint, and the velocity is filtered by a low pass filter with cutoff frequency of $20 \mathrm{~Hz}$. A 6-axis ATI load cell, which is attached to the tool plate of the Meka arm, is used for direct sensing of the tool force/torque wrench.

As shown in the first subplot of Fig. 2, the desired trajectory of the end-effector of the Meka arm is prescribed as a circle in the vertical plane, i.e., $x_{d}=$ $[0.125 \cos (t), 0.125 \sin (t)]$. The human desired path includes four lines with five ending points: $x_{h, 1}=[-0.063,0.104]^{T}$, $x_{h, 2}=[-0.185,0]^{T}, x_{h, 3}=[-0.063,-0.104]^{T}, x_{h, 4}=$ $[0,-0.095]^{T}$, and $x_{h, 5}=[0.063,-0.104]^{T}$, and an arc overlapping with the desired path of the end-effector of the Meka arm. Therefore, the tracking error is defined as below:

$e= \begin{cases}x-x_{d}, & t \leq 3.6 \mathrm{~s} \\ x-x_{h, 1}-\frac{\left(x_{h, 2}-x_{h, 1}\right)}{5.2}(t-3.6), & 3.6 \mathrm{~s}<t \leq 8.8 \mathrm{~s} ; \\ x-x_{h, 2}-\frac{\left(x_{h, 3}-x_{h, 2}\right)}{5.2}(t-8.8), & 8.8 \mathrm{~s}<t \leq 14.0 \mathrm{~s} ; \\ x-x_{h, 3}-\frac{\left(x_{h, 4}-x_{h, 3}\right)}{2.8}(t-14.0), & 14.0 \mathrm{~s}<t \leq 16.8 \mathrm{~s} ; \\ x-x_{h, 4}-\frac{\left(x_{h, 5}-x_{h, 4}\right)}{2.8}(t-16.8), & 16.8 \mathrm{~s}<t \leq 19.6 \mathrm{~s} ; \\ x-x_{d}, & t>19.6 \mathrm{~s} .\end{cases}$

Both robot and human desired paths are displayed on a monitor, as well as the actual trajectory. Impedance parameters in the impedance model (3) are $M_{d}=5 I_{2 \times 2}, C_{d}=750 I_{2 \times 2}$. The initial weights in the cost function (4) are: $Q_{1}=$ $10^{5} I_{2 \times 2}, Q_{2}=I_{2 \times 2}, R_{1}=0.001 I_{2 \times 2}$, and $R_{2}=0.01 I_{2 \times 2}$. 
The updating rate in the adaptation law (14) is $\alpha=10$. For the comparison purpose, we consider the following three conditions: 1) "robot leading" with $\left.r_{2} \equiv 0.01,2\right)$ "human leading" with $r_{2} \equiv 0.0001$, and 3) "adaptation" with $r_{2}(0)=$ 0.01 . To prevent $r_{2}$ from becoming too small or too large, we set it as 0.0001 when it is smaller than 0.0001 , and 0.01 when larger than 0.01 .

We conduct experiments with 10 subjects, who are told that there are 3 different experimental conditions but not what each condition means. For each condition, the subject is instructed to move the robot end-effector along the human desired path displayed on the monitor, and has the chance to practise until he/she feels confident of finishing the task. After the practice session, the subject performs 5 trials of the same experimental condition.

Results from representative trials are shown in Figs. 24. As shown in Fig. 2, the "adaptation" trajectory is close to the human desired path throughout the whole trial. In the "robot leading" condition, the end-effector cannot be moved to the leftmost point on human desired path, while the tracking error is relatively small when the robot and human desired paths coincide. These experimental results are largely coherent with the expectation. However, it is interesting to find that the "human leading" trajectory does not follow the human desired path well when the human desired path differs from the robot desired path. This is because during experiments, low damping in the human arm causes overshooting to occur frequently when moving the robot in the "human leading" condition. The tracking performance can be observed in the tracking error subplot of Fig. 3. In the force subplot of Fig. 3, it can be seen that larger forces are needed for the "robot leading" condition. Additionally, we show, in Fig. 4, the weight of the human contribution to the control sharing, $r_{2}^{-1}$, as well as the robot control gain $K_{1}$. Corresponding to the results in Fig. 3, the weight $r_{2}^{-1}$ increases when the subject increases the force, causing the gain $K_{1}$ to also become smaller. Conversely, when the subject decreases the force, the weight increases and so does the gain, indicating that the robot is regaining the leading role.

To understand the above results in a more intuitive way, we split the human desired path into 2 segments. The first segment is the portion where the human desired path differs from the robot desired path, as illustrated in the leftmost diagram of Fig. 5(a). The second segment is the portion where the human desired path overlaps with the robot desired path, as illustrated in the leftmost diagram of Fig. 5(b). We use the following measures to evaluate the performance of different methods along each segment and also along the entire path:

1) tracking error and interaction force along the first segment: $\mathcal{E}_{1}=\int_{3.6}^{19.6}\|e(t)\| d t$ and $\mathcal{F}_{1}=\int_{3.6}^{19.6}\|f(t)\| d t$

2) tracking error and interaction force along the second segment: $\mathcal{E}_{2}=\int_{0.0}^{3.6}\|e(t)\| d t+\int_{19.6}^{32.0}\|e(t)\| d t$ and $\mathcal{F}_{2}=$ $\int_{0.0}^{3.6}\|f(t)\| d t+\int_{19.6}^{32.0}\|f(t)\| d t$, and

3) tracking error, interaction force, and work done by sub-
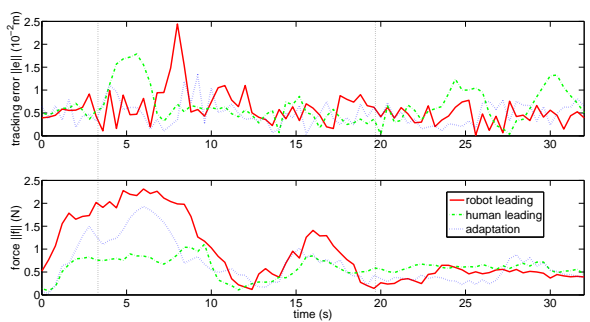

Fig. 3. Tracking error (top) and interaction force (bottom) for adaptive and fixed role cases. The two peaks in the interaction force correspond to the points in time where the subject wanted to lead the most.

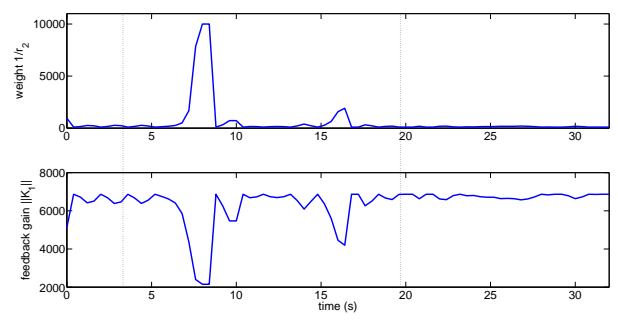

Fig. 4. Weight of interaction force (top) and robot control gain (bottom). The two peaks in each subplot correspond to the points in time where the subject wanted to lead the most.

ject along the complete path: $\mathcal{E}=\int_{0.0}^{32.0}\|e(t)\| d t, \mathcal{F}=$ $\int_{0.0}^{32.0}\|f(t)\| d t$, and $\mathcal{W}=\int_{0.0}^{32.0}\left\|f^{T}(t) \dot{x}(t)\right\| d t$.

As mentioned in the previous subsection, the mean and standard deviation of the above measures are calculated for $5 \times 10$ (trial number $\times$ user number) data points per setting. We investigate if there are any statistically significant differences between the 3 experimental conditions using oneway analysis of variance (ANOVA).

From Fig. 5(a), we find that the interaction force in the first segment for the "adaptation" and "human leading" conditions is significantly smaller than that for the "robot leading" condition, while tracking errors for all of the 3 conditions are similar. The tracking error in the second segment for the "adaptation" condition is significantly smaller than that for the "human leading" condition, as shown in Fig. 5(b). However, no significant difference is found in the interaction force in the second segment for all 3 conditions, because the robot and the human desired paths overlap, and there is no need for the subject to exert large forces. For measures involving the complete path, Fig. 5(c) shows that the tracking error for the "adaptation" and "robot leading" conditions is significantly smaller than that for the "human leading" condition, while the interaction force for the "adaptation" and "human leading" conditions is significantly smaller than that for the "robot leading" condition. Besides this, the work done $\mathcal{W}$ for the "adaptation" and "human leading" conditions is significantly smaller than that for the "robot leading" condition. Based on these results, we find that the "adaptation" condition achieves the best performance in that both human effort and trajectory tracking error are minimized, while the fixed-role conditions are limited by a performance trade-off between the 2 criteria. 

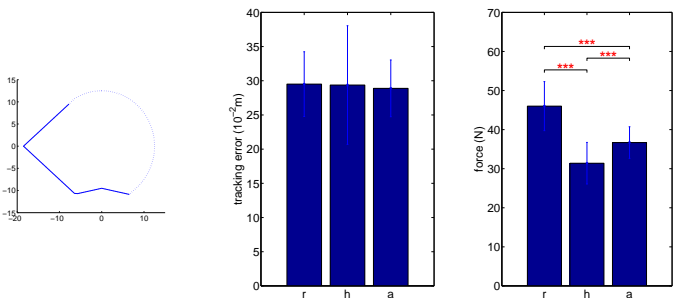

(a)
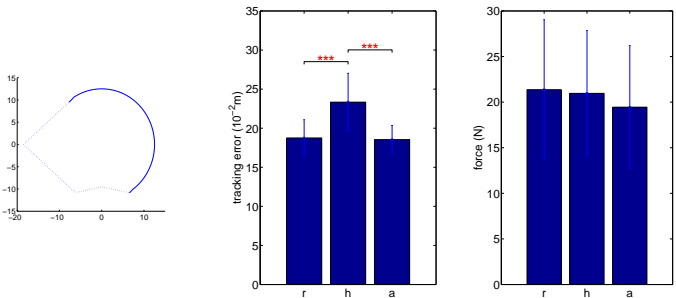

(b)
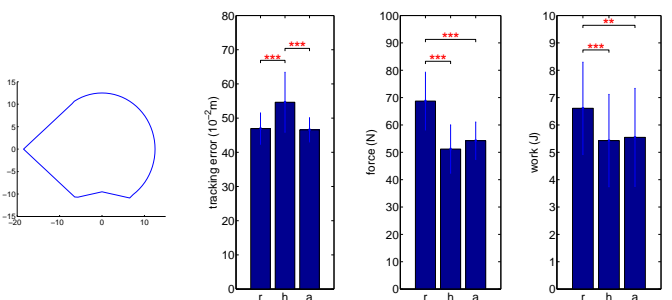

(c)

Fig. 5. Comparison of performance measures: (a) $\mathcal{E}_{1}\left(10^{-2} \mathrm{~m}\right)$ and $\mathcal{F}_{1}$ (N), (b) $\mathcal{E}_{2}\left(10^{-2} \mathrm{~m}\right)$ and $\mathcal{F}_{2}(\mathrm{~N})$, and $(\mathrm{c}) \mathcal{E}\left(10^{-2} \mathrm{~m}\right), \mathcal{F}(\mathrm{N})$, and $\mathcal{W}(\mathrm{J})$ for adaptive and fixed role cases. A double asterisk “**” indicates $p<0.01$, and a triple asterisk "***" $p<0.001$. The solid line in the leftmost diagram depicts the segment.

\section{CONCLUSIONS}

Human-robot collaboration has been investigated in this work, and formulated as a two-agent game. A role adaptation law based on game theory has been proposed, such that robot automatically adjusts its role when the human interaction force is changed. Through experimental studies, we have shown that role adaptation yields better overall performance, in the sense that both human effort and trajectory tracking error are minimized, than fixed-role interactions, which are limited by a trade-off between the two performance criteria.

\section{REFERENCES}

[1] Y. Li, K. Tee, S. S. Ge, and H. Li, "Building companionship through human-robot collaboration," in Social Robotics (G. Herrmann, M. Pearson, A. Lenz, P. Bremner, A. Spiers, and U. Leonards, eds.), vol. 8239 of Lecture Notes in Computer Science, pp. 1-7, Springer International Publishing, 2013.

[2] V. Duchaine and C. Gosselin, "Safe, stable and intuitive control for physical human-robot interaction," in Proceedings of the 2009 IEEE International Conference on Robotics and Automation, (Kobe, Janan), pp. 3676-3681, 2009.

[3] S. S. Ge, C. Yang, Y. Li, and T. H. Lee, "Decentralized adaptive control of a class of discrete-time multi-agent systems for hidden leader following problem," in Proceedings of the IEEE/RSJ International
Conference on Intelligent Robots and Systems (IROS), (St. Louis, Missouri), pp. 5065-5070, October 11-15 2009.

[4] Y. Yamamoto and X. Yun, "Effect of the dynamic interaction on coordinated control of mobile manipulators," IEEE Transactions on Robotics and Automation, vol. 12, no. 5, pp. 816-824, 1996.

[5] R. Chipalkatty, G. Droge, and M. B. Egerstedt, "Less is more: Mixed-initiative model-predictive control with human inputs," IEEE Transactions on Robotics, vol. 29, no. 3, pp. 695-703, 2013.

[6] N. Hogan, "Impedance control: an approach to manipulation-Part I: Theory; Part II: Implementation; Part III: Applications," Journal of Dynamic Systems, Measurement, and Control, vol. 107, no. 1, pp. 124, 1985.

[7] Y. Li and S. S. Ge, "Human-robot collaboration based on motion intention estimation," IEEE/ASME Transactions on Mechatronics, vol. 19, no. 3, pp. 1007-1014, 2014.

[8] H. Wang and K. Kosuge, "Attractor design and prediction-based adaption for a robot waltz dancer in physical human-robot interaction," in Proceedings of the 10th World Congress on Intelligent Control and Automation, pp. 3810-3815, 2012.

[9] K. P. Tee, R. Yan, and H. Li, "Adaptive admittance control of a robot manipulator under task space constraint," in Proceedings of the 2010 IEEE International Conference on Robotics and Automation, pp. 5181-5186, 2010.

[10] N. Jarrasse, V. Sanguineti, and E. Burdet, "Slaves no longer: review on role assignment for human-robot joint motor action," Adaptive Behavior, vol. 22, no. 1, pp. 70-82, 2014.

[11] J. R. Medina, D. Lee, and S. Hirche, "Risk-sensitive optimal feedback control for haptic assistance," in Proceedings of the 2012 IEEE International Conference on Robotics and Automation, pp. 1025-1031, 2012.

[12] M. Saida, J. R. Medina, and S. Hirche, "Adaptive attitude design with risk-sensitive optimal feedback control in physical human-robot interaction," in Proceedings of the 21st IEEE International Symposium on Robot and Human Interactive Communication, (Paris, France), pp. 955-961, September 2012.

[13] J. R. Medina, M. Lawitzky, A. Molin, and S. Hirche, "Dynamic strategy selection for physical robotic assistance in partially known tasks," in Proceedings of the 2013 IEEE International Conference on Robotics and Automation, pp. 1180-1186, 2013.

[14] A. Mortl, M. Lawitzky, A. Kucukyilmaz, M. Sezgin, C. Basdogan, and S. Hirche, "The role of roles: Physical cooperation between humans and robots," The International Journal of Robotics Research, vol. 31, no. 13, p. 16561674, 2012.

[15] Z. Wang, A. Peer, and M. Buss, "An hmm approach to realistic haptic human-robot interaction," in Proceedings of the Third Joint Eurohaptics Conference and Symposium on Haptic Interfaces for Virtual Environment and Teleoperator Systems, pp. 374-379, 2009.

[16] P. Evrard and A. Kheddar, "Homotopy switching model for dyad haptic interaction in physical collaborative tasks," in Proceedings of the 3rd Joint EuroHaptics Conference and Symposium on Haptic Interfaces for Virtual Environment and Teleoperator Systems, (Salt Lake City, Utah, USA), pp. 45-50, 2009.

[17] N. Jarrasse, T. Charalambous, and E. Burdet, "A framework to describe, analyze and generate interactive motor behaviors," PLoS ONE, vol. 7, no. 11, p. e49945, 2013.

[18] S. P. Buerger, Stable, high-force, low-impedance robotic actuators for human-interactive machines. $\mathrm{PhD}$ thesis, MIT, Department of Mechanical Engineering, 2005.

[19] J. Kober and J. Peters, "Policy search for motor primitives in robotics," Machine Learning, vol. 84, pp. 171-203, 2011.

[20] T. Shima and S. Rasmussen, eds., UAV Cooperative Decision and Control. Society for Industrial and Applied Mathematics, 2009.

[21] G. Hudas, K. G. Vamvoudakis, D. Mikulski, and F. L. Lewis, "Online adaptive learning for team strategies in multi-agent systems," The Journal of Defense Modeling and Simulation: Applications, Methodology, Technology, vol. 9, no. 1, pp. 59-69, 2012.

[22] S. S. Ge, Y. Li, and C. Wang, "Impedance adaptation for optimal robotenvironment interaction," International Journal of Control, vol. 87, no. 2, pp. 249-263, 2014.

[23] C. Yang, G. Ganesh, S. Haddadin, S. Parusel, A. Albu-Schaeffer, and E. Burdet, "Human-like adaptation of force and impedance in stable and unstable interactions," IEEE Transactions on Robotics, vol. 27, no. 5, pp. 918-930, 2011.

[24] J. E. Slotine and W. Li, Applied Nonlinear Control. Englewood Cliff, NJ: Prentice-Hall, Inc., 1991. 